Patrushev A. V. ${ }^{1}$, Ostanina T. N. ${ }^{1}$, Rudoy V. M. ${ }^{1}$, Vereshchagina A. V. ${ }^{1}$, Zalesova O. L. ${ }^{2}$, Soloviev A. S. ${ }^{2}$, Shtirba N. I. ${ }^{2}$

\title{
The choice of the conditions to receive the electrolytic zinc powders for metal-rich compositions*
}

\author{
${ }^{1}$ Ural Federal University, \\ 19 Mira street, 620002 Ekaterinburg \\ ${ }^{2}$ Scientifically Industrial Holding VMP \\ Closed Joint Stock Company, \\ 105 Amundsen street, 620016 Ekaterinburg
}

The paper presents a method of obtaining high-dispersed zinc powders by electrolysis and comparison of the properties of zinc-rich compositions prepared using as a pigment zinc powders obtained by different methods. Measurements have shown that the electrical conductivity of zinc-rich coatings containing electrolytic zinc powder, not inferior to the conductivity of the film with powder PZHD-0 obtained by the method of evaporation-condensation, despite the significant difference in the amount of zinc pigment. On the basis of the received data we can conclude that the use of electrolytic zinc powder as a pigment will significantly save zinc.

*This work was supported by RFBR, project number 11-03-00226

(c) Patrushev A. V., Ostanina T. N., Rudoy V. M., Vereshchagina A. V., Zalesova 0. L.,Soloviev A. S., Shtirba N. I., 2015

\section{Introduction}

The main structural material of modernity is steel, which actively corrodes and therefore requires anticorrosive protection. For the prevention of destruction of metal structures, passive defense is used: the surface is covered by various isolating coatings: bituminous mastics, polyethylene and epoxy film, and isolation. However, in places where the integrity of such coating is broken, metal active corrodes. In contrast to the isolating coatings, tread zinc-rich coatings possess complex mechanism of protective ac- tions. With the penetration of moisture into the film pigment, zinc powder acts as protector, providing a cathode-term protection of steel substrates. Currently, the production of zinc-rich compositions uses zinc powder, which is produced by the evaporation-condensation (1). The powder particles obtained by this method have a spherical shape and size of 3-8 micron (2).

Prerequisite of zinc-rich coating effectiveness is its conductivity (3) that occurs with the introduction of a large amount of 
pigment to the composites. Substitution of zinc particles of spherical form with 'scaly' ones enhances conductivity in coatings. Electrolytic zinc powders are without doubt advantageous in terms of the properties of the pigment, as they are characterized by a branched structure and high degree of purity of the metal. However, the mean particle size after zinc removal from the cathode is substantially higher than that of powders obtained by metallurgical method; therefore, there requires an additional operation for grinding dendritic precipitation.

The advantage of electrolytic method of producing dendritic metal precipitation is the ability to influence the dynamic of growth and structure of the particles, changing the composition of the solu-

\section{The experimental procedure}

To select the conditions for obtaining a high-dispersed zinc powder, studies of the dynamics of growth of dendritic percipitation of zinc in solutions of different concentrations $(0.12,0.24,0.36$ and 0.45 $\mathrm{mol} / 1 \mathrm{ZnO}$, and 4 mole / $1 \mathrm{NaOH}$ ) at the depletion rate (Dr) 3, 6, 9 were carried out. Depletion factor Dr is the ratio of a given current to the limit one on a smooth electrode. Investigations were carried out at the facility, which allows simultaneous recording of the amount of capacity, conducting videorecording of percipitation growth and determining the amount of generated hydrogen. Cylindrical rod cathode made from zinc with diameter of 1.8 $\mathrm{mm}$ was put in the cell center. The edge of the cell was covered with anode from zinc tion, the amount of current or potential, or polarization mode. In industry, powder precipitation is obtained under conditions of polarization by constant current exceeding the value of the limiting diffusion current. Quantitative description of the process of electrocrystallisation of metal dendrites using model representations provides the opportunity for reasonable choice of the conditions for obtaining powder precipitation with specified characteristics (4).

The aim of this study was in obtaining high-dispersed dendritic zinc precipitation and comparing the properties of zincrich compositions prepared using zinc as pigment powders obtained by different methods.

sheet. Electrochemical measurements were performed using an electrochemical station ZIVESP5; Sony DSR-200SE4 was used for video-recording of the development of dendrites.

Experimental batch of zinc powder was obtained in laboratory electrolysis unit by setting a DC. After powder trimmings from the cathode, powder was washed off the electrolyte, dried and milled in a porcelain mortar. For a comparative study of conductivity of zinc-rich coatings, compositions were prepared using powders produced by electrolysis and evaporation-condensation method. Films resistance was defined by the currentvoltage curves (3).

\section{Experiment results}

Intensive growth of dendrites in the initial stages of electrodeposition was gradually slowing down. Termination of active extension of dendrites is due to a 
decrease of the current density amid the growth to the limiting diffusion, which confirms a sharp decrease in the absolute value of the polarization observed in the chronopotentiograms, and termination of hydrogen gas emission. Significant impact on the dynamics of dendritic precipitation is played by electrodeposition conditions. Increasing the current and the concentration of the solution increases the rate of elongation of precipitation with simultaneous reduction in time of active growth of dendrites. Maximum length of dendritic precipitation with increasing $\mathrm{Dr}$ is growing, but is little affected by concentration. Differential (instantaneous) current output, which was calculatedfrom the change of the amount of emitted hydrogen in time period $\Delta t$, is of great importance for the exploration of the kinetics of electrodeposition of dendrite metal precipitations. Differential current output increases with decreasing of given current and with increase in the concentration of metal ions in solution. At the end of the elongation of the active precipitation, its value is close to 1 .

\section{Model description}

For selection of the conditions for obtaining high-dispersed zinc powders, structural properties of growing dendrite precipitation were calculated: the tip radius of RB branches of dendrites and the density of their placement amid the growth of $\mathrm{N}$ percipitation using model representations [3].

Analysis of the experimental data has proved that the change in dendrites length in time (t) can be described with high level of accuracy with exponential relationship

$$
y(t)=y_{0}\left(1-\exp \left(-\frac{t}{\tau}\right)\right),
$$

where yo and $\tau$ are empirical parameters.

For approximation of the change in differential current output, empirical relationship of the following form can be used:

$$
B_{T}(t)=\frac{a+b \cdot t}{c+d \cdot t} .
$$

When receiving the dendritic precipitations, crystallization of metal occurs, primarily, on tops of dendrites generating the precipitation growth front. The cur- rent density on the tops can be calculated from the equation of mixed kinetics (4):

$$
\frac{1}{i_{B}}=\frac{1}{i_{\text {кин }}}+\frac{1}{i_{\text {сф }}} \text {. }
$$

At high cathodic polarization kinetic current (ikin) is much larger than the diffusion (isf), so in equation (3), the first addend can be neglected. Then it can be written for the current density on the tops of the dendrites:

$$
i_{B}(t)=i_{\mathrm{c} \phi}(t)=\frac{z F D c_{0}}{r_{B}(t)},
$$

where: $D$ is the diffusion coefficient of zinc ions, $c_{0}$ is the concentration of zinc ions in the solution.

In accordance with Faraday's law the rate of precipitation elongation is proportional to current density on the tops:

$$
i_{B}(t)=\frac{d y}{d t} \cdot \frac{z F}{v_{M e}},
$$

where $v_{M e}=\frac{A_{M e}}{\rho_{M e}}$ is the molar metal vol- 
ume. The joint solution of the equations (4) and (5), and differentiation of dependence of the length of the dendrites from the time (1) allowed to calculate the radius of tops:

$$
r_{B}(t)=\frac{D c_{o} v_{M e} \tau}{y_{0} \exp \left(-\frac{t}{\tau}\right)},
$$

Some part of the current goes onto the reduction of the metal; this part is derived from the magnitude of instant current output (2):

$$
I \cdot B m(t)=i_{B} 2 \pi r_{B}^{2} \cdot N \cdot \pi\left(d_{0}+2 y\right) H .
$$

From equation (7) with (1) and (5) we have obtained an expression for the calculation of density of peaks layout peaks at the growth front:

$$
N=\frac{I \cdot B m(t) \cdot\left(v_{M e} / z F\right)}{r_{B}^{2}\left(\frac{y_{0}}{\tau}\right) \exp \left(-\frac{t}{\tau}\right) 2 \pi^{2}\left(d_{0}+2 y(t)\right) H}
$$

The calculations showed that during electrolysis an increase of the dendrites peaks radius (Figure 1), and a decrease in their number. Increase in depletion rate (values of given current) and decrease in the concentration of the solution help to ensure receiving of a dendrite with a small peaks radius.

Based on these results the following electrolysis conditions were selected to receive an experimental batch of zinc powder: zinc ion concentration of 0.1 mol $/ 1, \operatorname{Dr}=9$. Deposition was led on copper cylindrical electrodes with diameter of $11 \mathrm{~mm}$ and height of $40 \mathrm{~mm}$, which were beforehand covered with a zinc layer with thickness of 20 microns. Comparative studies of the properties of electrolytic zinc powder and powder of brand PTSDV-0 prepared by evapora- tion-condensation method, as well as the properties of zinc-rich coatings prepared using these powders were carried out on the CJS Scientifically Industrial Holding VMP. It was established that oil-absorption of PTSVD-0 powder is 5 times less than oil absorption of zinc powder obtained by electrolysis, which indicates a high specific surface of the latter. The consequence of this is that the use of a pigment of electrolytic zinc powder leads to a significant reduction in the critical pigment volume fraction (CPVC)) in the paint (Table. One).

Table 1

Properties of zinc powders, obtained by different methods

\begin{tabular}{c|c|c} 
Parameter & $\begin{array}{c}\text { Electrolytic } \\
\text { powder }\end{array}$ & PTSVD-0 \\
\hline $\begin{array}{c}\text { Oil absorption } \\
\mathrm{Mg} / 100 \mathrm{~g}\end{array}$ & 65,8 & 12,6 \\
\hline $\mathrm{CPVC} \%$ & 25,3 & 28,0
\end{tabular}

The measurements showed that the conductivity of zinc-rich coatings containing an electrolytic zinc powder is not inferior to the conductivity of the film with a powder PTSVD-0, despite the significant difference in the amount of zinc pigment. Comparison of protective properties of zinc-rich films requires addition-

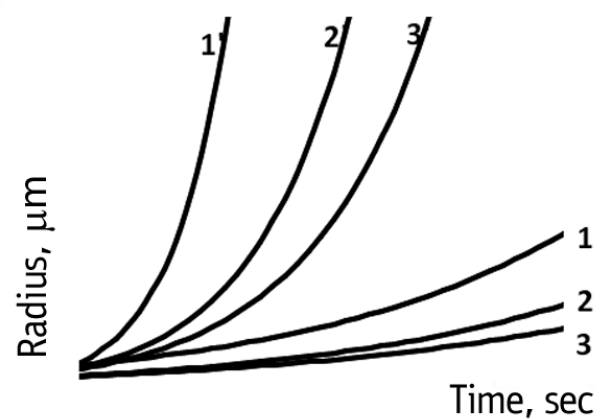

Fig.1. Time variation of the radius of the peaks of zinc dendrites at a concentration $0,12 \mathrm{~mol} / \mathrm{L}(1,2,3)$ and $0,24 \mathrm{~mol} / \mathrm{L}$ $\left(1^{\prime}, 2^{\prime}, 3^{\prime}\right)$ and $\operatorname{Dr} 3$ (1,1'), 6 (2 2 '), 9 (3,3’) 
al investigation; however, based on these electrolytic zinc powder as a pigment aldata we can conclude that the usage of the lows cutting costs.

1. Subbotina O. Yu., Pirogov V. D., Samsonova A. I., Balahnina L. A. Compounded coating ZVES and ZINOL for protection from corrosion at ship building and ship repair. Varnish-and-paint Materials And Their Use. 1998; 9:25-30. [Google Scholar].

2. Frishberg I. V., Yurkina L. P., Subbotina O. Yu., PosohinYu. P. Modern domestic Zncompounded paints. Practice of their use. Varnish-and-paint Materials And Their Use. 1997; 12:8-13. [Google Scholar].

3. Rudoy V. M., Yaroslavzeva O. V., Ostanina T. N., Yurkina L. P., Subbotina O. Yu. Electroconductivity of Me-compounded polymer compositions. Protection of Metals. 1998; 3(5):527-532. [Google Scholar].

4. Darinzeva A. B., Patrushev A. V., Ostanina T. N., Malkov V. B. Electrocristallzation of dendritic precipitates of $\mathrm{Zn}$ and $\mathrm{Ni}$ at the galvanostatistic conditions. Vestnik Kazanskogo Technologicheskogo Universiteta. 2012; 15(B19):62-66. [Google Scholar]. 
А. В. Патрушев ${ }^{1}$, Т. Н. Останина ${ }^{1}$, В. М. Рудой ${ }^{1}$, А. В. Верещагина ${ }^{1}$, О. Л. Залесова ${ }^{2}$, А. С. Соловьев ${ }^{2}$, Н. И. Штырба ${ }^{2}$

${ }^{1}$ Уральский федеральный университет, 620002, Екатеринбург, ул. Мира, 19.

23 ЗА НПХ ВМП, 620016, Екатеринбург, ул. Амундсена, 105

\section{Выбор условий получения электролитических порошков цинка для металлнаполненных композиций*}

В работе представлен способ получения высокодисперсных порошков цинка электролизом и сравнение свойств цинкнаполненных композиций, приготовленных с применением в качестве пигмента цинковых порошков, полученных разными методами. Проведенные измерения показали, что удельная электропроводность цинкнаполненных покрытий, содержащих электролитический порошок цинка, не уступает по проводимости пленкам с порошком ПЦВД-0, полученного методом испарения-конденсации, несмотря на существенное различие в количестве цинкового пигмента. На основании полученных данных можно сделать вывод, что использование электролитического порошка цинка в качестве пигмента позволит значительно экономить цинк.

* Работа выполнена при поддержке РФФИ, проект № 11-03-00226

(C) Патрушев А. В., Останина Т. Н., Рудой В. М., Верещагина А. В., Залесова 0. Л., Соловьев А. С., Штырба Н. И., 2015

\section{Введение}

Основной конструкционный материал современности - сталь, которая активно подвергается коррозии и поэтому нуждается в противокоррозионной защите. Для предотвращения разрушения металлических сооружений используют пассивную защиту на поверхность наносят различные изолирующие покрытия: битумные мастики, полиэтиленовую, эпоксидную и пленочную изоляции. Однако в местах нарушения целостности такого покрытия металл активно корродирует. В отличие от изолирующих протекторные цинкнаполненные покрытия обладают комплексных механизмом защитного действия. При проникновении влаги в пленку пигмент - цинковый порошок выступает в роли протектора, обеспечивая катодную защиту стальной основы. 
В настоящее время при производстве цинкнаполненных составов используют цинковый порошок, который получают методом испарения-конденсации [1]. Частицы порошка, полученные данным методом, имеют сферическую форму и размер 3-8 мкм [2].

Обязательным условием эффективности цинкнаполненных покрытий является электропроводность [3], которая возникает при введении в состав композитов большого количества пигмента. Замена части частиц цинка сферической формы на чешуйчатую способствует повышению проводимости покрытий.

Несомненным преимуществом с точки зрения свойств пигмента обладают электролитические порошки цинка, так как они характеризуются разветвленной структурой и высокой степенью чистоты металла. Однако средний размер частиц цинка после съема с катода существенно выше, чем у порошков, полученных металлургическим методом, поэтому требуется дополнительная операция по размолу дендритных осадков.

Достоинством электролитического метода получения дендритных осадков металла является возможность влиять на динамику роста и структуру частиц, изменяя состав раствора, величину тока или потенциала, режим поляризации. В промышленности порошкообразные осадки получают в условиях поляризации постоянным током, превышающим величину предельного диффузионного тока. Количественное описание процесса электрокристаллизации дендритов металлов с помощью модельных представлений позволяет обоснованно выбирать условия получения порошкообразных осадков с заданными свойствами [4].

Цель настоящей работы состояла в получении высокодисперсных дендритных осадков цинка и сравнении свойств цинкнаполненных композиций, приготовленных с применением в качестве пигмента цинковых порошков, полученных разными методами.

\section{Методика эксперимента}

Для выбора условий получения высокодисперсного порошка цинка были проведены исследования по изучению динамики роста дендритных осадков цинка в растворах разной концентрации $(0,12 ; 0,24 ; 0,36$ и 0,45 моль/л $\mathrm{ZnO}$ и 4 моль/л $\mathrm{NaOH}$ ) при коэффициентах истощения (Ки) 3, 6, 9. Коэффициент истощения Ки - отношение заданного тока к предельному на гладком электроде. Исследования проводили на установке, позволяющей одновременно регистрировать величину потенциала, проводить видеозапись роста осадка и определять объем выделяю- щегося водорода. В центр цилиндрической ячейки подводили стержневой катод, выполненный из цинка диаметром 1,8 мм. По краю ячейки располагали анод из цинкового листа. Электрохимические измерения проводили с помощью электрохимической станции ZIVESP5, для видеозаписи процесса развития дендритов использовали видеокамеру Sony DSR-200SE [4].

Опытную партию цинкового порошка получали в лабораторном электролизере при задании постоянного тока. После счистки с катода порошок отмывали от электролита, сушили и 
измельчали в фарфоровой ступке. Для сравнительного исследования электропроводности цинкнаполненных покрытий были приготовлены составы с применением порошков, полученных электролизом и методом испаренияконденсации. Сопротивление пленок определяли по вольтамперным кривым [3].

\section{Результаты экспериментов}

Интенсивный рост дендритов на начальных стадиях электроосаждения постепенно замедлялся. Прекращение активного удлинения дендритов связано со снижением плотности тока на фронте роста до предельной диффузионной, что подтверждает резкое уменьшение абсолютной величины поляризации, наблюдаемое на хронопотенциограммах, и прекращение выделения газообразного водорода. Существенное влияние на динамику развития дендритного осадка оказывают условия электроосаждения. Повышение тока и концентрации раствора приводит к увеличению скорости удлинения осадка с одновременным уменьшением времени активного роста дендритов. Предельная длина дендритного осадка с увеличением Ки растет, но мало зависит от концентрации. Большое значение для исследования кинетики электроосаждения дендритных осадков металлов имеет дифференциальный (мгновенный) выход по току, который рассчитывали по изменению объема выделившегося водорода за интервал времени $\Delta t$. Дифференциальный выход по току возрастает с уменьшением заданного тока и увеличением концентрации ионов металла в растворе. В момент окончания активного удлинения осадка его величина приближается к единице.

\section{Модельное описание}

Для выбора условий получения высокодисперсных порошков цинка был проведен расчет структурных свойств растущего дендритного осадка: радиуса вершин ветвей дендритов $r_{\text {в }}$ и плотности их размещения на фронте роста осадка N с помощью модельных представлений [3].

Анализ экспериментальных данных показал, что изменение длины дендритов во времени $(t)$ с высокой степенью точности может быть описано экспоненциальной зависимостью

$$
y(t)=y_{0}\left(1-\exp \left(-\frac{t}{\tau}\right)\right),
$$

Для аппроксимации изменения дифференциального выхода по току может быть использована эмпирическая зависимость вида:

$$
B_{T}(t)=\frac{a+b \cdot t}{c+d \cdot t} .
$$

При получении дендритных осадков кристаллизация металла протекает преимущественно на вершинах дендритов, образующих фронт роста осадка. Плотность тока на вершинах может быть рассчитана по уравнению смешанной кинетики [4]:

$$
\frac{1}{i_{B}}=\frac{1}{i_{\text {кин }}}+\frac{1}{i_{\text {сф }}} .
$$

где $y_{0}$ и $\tau$ - эмпирические параметры. 
При высокой катодной поляризации кинетический ток $\left(i_{\text {кин }}\right)$ много больше, чем диффузионный $\left(\mathrm{i}_{\text {сф }}\right)$, поэтому в уравнении (3) первым слагаемым можно пренебречь. Тогда для плотности тока на вершинах дендритов можно записать:

$$
i_{B}(t)=i_{\mathrm{c} \phi}(t)=\frac{z F D c_{0}}{r_{B}(t)},
$$

$D$ - коэффициент диффузии ионов цинка, $c_{0}-$ концентрация ионов цинка в растворе.

В соответствии с законом Фарадея скорость удлинения осадка пропорциональна плотности тока на вершинах:

$$
i_{B}(t)=\frac{d y}{d t} \cdot \frac{z F}{v_{M e}}
$$

где $v_{M e}=\frac{A_{M e}}{\rho_{M e}}-$ мольный объем металла. Совместное решение уравнений (4) и (5) и дифференцирования зависимости длины дендритов от времени (1) позволило рассчитать радиус вершин:

$$
r_{B}(t)=\frac{D c_{o} v_{M e} \tau}{y_{0} \exp \left(-\frac{t}{\tau}\right)} .
$$

На восстановление металла идет часть тока, определяемая величиной мгновенного выхода по току (2):

$$
I \cdot B m(t)=i_{B} 2 \pi r_{B}^{2} \cdot N \cdot \pi\left(d_{0}+2 y\right) H .
$$

Из уравнения (7) с учетом (1) и (5) получили выражение для расчета плотности расположения вершин на фронте роста:

$$
N=\frac{I \cdot B m(t) \cdot\left(v_{M e} / z F\right)}{r_{B}^{2}\left(\frac{y_{0}}{\tau}\right) \exp \left(-\frac{t}{\tau}\right) 2 \pi^{2}\left(d_{0}+2 y(t)\right) H} .
$$

Проведенные расчеты показали, что в процессе электролиза происходит увеличение радиуса вершин дендритов (рис. 1) и уменьшение их числа. Увеличение коэффициент истощения (величины заданного тока) и уменьшение концентрации раствора способствует получению дендритов с малым радиусом вершин.

На основе полученных результатов были выбраны следующие условия электролиза для получения опытной партии цинкового порошка: концентрация ионов цинка 0,1 моль/л, Ки $=9$. Осаждение вели на медных цилиндрических электродах диаметром 11 мм и высотой 40 мм, на которые предварительно наносили слой цинка толщиной 20 мкм.

На предприятии ЗАО НПХ ВМП были проведены сравнительные исследования свойств электролитического порошка цинка и порошка марки ПЦДВ-0, полученного методом испарения-конденсации, а также свойств цинкнаполненных лакокрасочных покрытий, приготовленных с применением этих порошков. Установлено, что

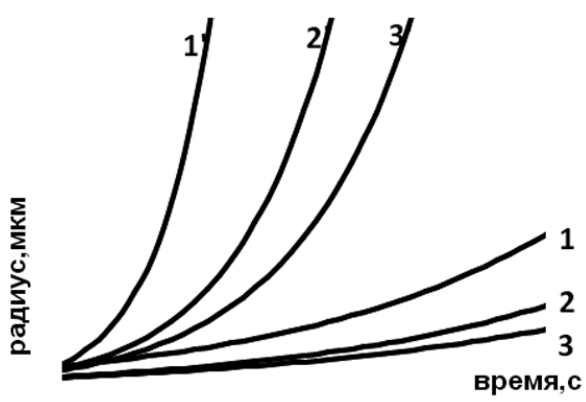

Рис. 1. Изменение во времени радиуса вершин дендритов цинка при концентрации 0,12 моль/л $(1,2,3)$ и 0,24 моль/л (1',2',3') и Ки 3(1,1'), 6(2,2'), $9\left(3,3^{\prime}\right)$ 
маслоемкость порошка марки ПЦВД-0 в 5 раз меньше маслоемкости цинкового порошка, полученного электролизом, что свидетельствует о большой удельной поверхности последнего. Следствие этого использование в качестве пигмента электролитического порошка цинка приводит к существенному снижению критической объемной доли пигмента (КОКП) в краске (табл. 1).

Проведенные измерения показали, что удельная электропроводность цинкнаполненных покрытий, содержащих электролитический порошок цинка, не уступает по проводимости пленкам с порошком ПЦВД-0 несмотря на существенное различие в количестве цинкового пигмента. Для сравнения протекторных свойств цинкнаполненных пленок необходимы дополнительные исследования, однако на основании полученных данных можно сделать вывод, что использование электролитического порошка цинка в качестве пигмента позволит значительно экономить цинк.

Таблица 1

Свойства цинковых порошков, полученных разными методами

\begin{tabular}{l|c|c}
\multicolumn{1}{c|}{ Параметр } & Порошок электролитический & ПЦВД-0 \\
\hline Маслоемкость Мг/100 г & 65,8 & 12,6 \\
\hline КОКП \% & 25,3 & 28,0
\end{tabular}

1. Субботина О. Ю., Пирогов В. Д., Самсонова А. И., Балахнина Л. А. Наполненные покрытия ЦВЭС и ЦИНОЛ для защиты от коррозии в судостроении и судоремонте // Лакокрасочные материалы и их применение. 1998. № 9. С. 25-30.

2. Фришберг И. В., Юркина Л. П., Субботина О. Ю., Посохин Ю. П. Современные отечественные цинкнаполненные краски. Опыт их применения // Лакокрасочные материалы и их применение. 1997. № 2. С. 8-13.

3. Рудой В. М., Ярославиева О. В., Останина Т. Н., Юркина Л. П., Субботина О. Ю. Электропроводность металлнаполненных полимерных композиций // Защита металлов. 1998. Т. 34, № 5. С. 527-532.

4. Даринцева А. Б., Патрушев А. В., Останина Т. Н., Малков В. Б. Электрокристаллизация дендритных осадков цинка и никеля в гальваностатических условиях // Вестн. Казан. технолог. ун-та. 2012. Т. 15. В. 19. С. 62-66. 